\title{
Erratum to: The JUPITER Trial: Myth or Reality?
}

\author{
Ryan P. Morrissey • George A. Diamond • Sanjay Kaul
}

Published online: 20 August 2011

(C) Springer Science+Business Media, LLC 2011

\section{Erratum to: Curr Atheroscler Rep}

\section{DOI 10.1007/s11883-011-0197-9}

This article was published in the October 2011 issue of Current Atherosclerosis Reports (volume 13, issue 5). On page 417, in the second paragraph of the second column, the phrase "CV mortality" should be "all-cause mortality".

The online version of the original article can be found at http://dx.doi. org/10.1007/s11883-011-0197-9.

R. P. Morrissey

Cedars-Sinai Heart Institute, Cedars-Sinai Medical Center,

Los Angeles, CA 90048, USA

e-mail: ryan.morrissey@cshs.org

G. A. Diamond $\cdot$ S. Kaul $(\square)$

Division of Cardiology, Cedars-Sinai Medical Center,

8700 Beverly Blvd,

Los Angeles, CA 90048, USA

e-mail:kaul@cshs.org

G. A. Diamond

e-mail: gadiamond@pol.net 